\title{
An Overview of Nonstandard Signals in Cosmological Data ${ }^{\dagger}$
}

\author{
George Alestas $\ddagger, *(\mathbb{D}$, George V. Kraniotis $\ddagger(\mathbb{D}$ and Leandros Perivolaropoulos $\ddagger$ (D) \\ Division of Theoretical Physics, University of Ioannina, 45110 Ioannina, Greece; gkraniotis@uoi.gr (G.V.K.); \\ leandros@uoi.gr (L.P.) \\ * Correspondence: g.alestas@uoi.gr \\ + Presented at the 1st Electronic Conference on Universe, 22-28 February 2021; Available online: \\ https://ecu2021.sciforum.net/. \\ $\ddagger$ Current address: Department of Physics, University of Ioannina, 45110 Ioannina, Greece.
}

check for

updates

Citation: Alestas, G.; Kraniotis, G.V.; Perivolaropoulos, L. An Overview of Nonstandard Signals in Cosmological Data. Phys. Sci. Forum 2021, 2, 28. https://doi.org/10.3390/ECU202109333

Academic Editor: Anupam

Mazumdar

Published: 25 February 2021

Publisher's Note: MDPI stays neutral with regard to jurisdictional claims in published maps and institutional affiliations.

Copyright: (c) 2021 by the authors. Licensee MDPI, Basel, Switzerland. This article is an open access article distributed under the terms and conditions of the Creative Commons Attribution (CC BY) license (https:// creativecommons.org/licenses/by/ $4.0 /)$.

\begin{abstract}
We discuss in a unified manner many existing signals in cosmological and astrophysical data that appear to be in some tension ( $2 \sigma$ or larger) with the standard $\Lambda$ CDM as defined by the Planck18 parameter values. The well known tensions of $\Lambda \mathrm{CDM}$ include the $H_{0}$ tension the $S_{8}$ tension and the lensing $\left(A_{\text {lens }}\right) \mathrm{CMB}$ anomaly. There is however, a wide range of other, less standard signals towards new physics. Such signals include, hints for a closed universe in the CMB, the cold spot anomaly indicating non-Gaussian fluctuations in the $\mathrm{CMB}$, the hemispherical temperature variance assymetry and other $\mathrm{CMB}$ anomalies, cosmic dipoles challenging the cosmological principle, the Lyman- $\alpha$ forest Baryon Accoustic Oscillation anomaly, the cosmic birefringence in the CMB, the Lithium problem, oscillating force signals in short range gravity experiments etc. In this contribution present the current status of many such signals emphasizing their level of significance and referring to recent resources where more details can be found for each signal. We also briefly mention some possible generic theoretical approaches that can collectively explain the non-standard nature of these signals. In many cases, the signals presented are controversial and there is currently debate in the literature on the possible systematic origin of some of these signals. However, for completeness we refer to all the signals we could identify in the literature citing also references that dispute their physical origin.
\end{abstract}

Keywords: cosmological data; anomalies; cosmic dipoles; lithium problem; cosmic birefrigence; $\mathrm{CMB}$ anomalies; $H_{0}$ problem; growth tension; LCDM standard model; $S_{8}$ tension

\section{Introduction}

We are experiencing an era where a single cosmological model is heralded by experts as the gold standard in explaining the way the universe behaves at a large scale. This model is $\Lambda \mathrm{CDM}$ and contains cold dark matter as well as a cosmological constant associated with dark energy. Despite the huge improvements of the cosmological observations that have been made over the last years, $\Lambda C D M$ seems to still be very consistent with most of the data produced [1-12]. However, this has been the case only for the majority and not the entirety of these data. There is arguably significant evidence, now more than ever, that the originally thought negligible imperfections of $\Lambda \mathrm{CDM}$ are actually deep cracks that indicate underlying pathologies of the model.

In this light, we attempt to discuss in a unified manner many existing signals in cosmological and astrophysical data that appear to be in some tension ( $2 \sigma$ or larger) with the standard $\Lambda C D M$ model as defined by the Planck18 parameter values. In addition to the well known tensions ( $H_{0}$ tension, $S_{8}$ tension and $A_{\text {lens }}$ anomaly), there is a wide range of other less discussed, less-standard signals at a lower statistical significance level than the $H_{0}$ tension which may also constitute hints towards new physics. The goal of this manuscript is to collectively present the current status of these signals and their level of significance, refer to recent sources where more details can be found for each signal and discuss possible generic theoretical approaches that can collectively explain their non-standard nature. 
In order to access the significance of each non-standard signal as well as the possibility that it can lead to new physics one must answer the following questions/points of study:

- What are the current cosmological and astrophysical datasets that include such nonstandard signals?

- What is the statistical significance of each signal?

- Is there a common theoretical framework that may explain these non-standard signals if they are of physical origin?

There have been previous similar studies [13,14] collecting and discussing signals in data that are at some statistical level in tension with the standard $\Lambda$ CDM model, but these are by now outdated. This manuscript serves as an attempt to provide an updated collection of these non-standard signals with emphasis to more recent measurements which may prove to be a useful resource for the community.

\section{A Collection of Non-Standard Signals}

In this section, we attempt to provide an extensive list of the non-standard cosmological signals in cosmological data. In many cases the signals are controversial and there is currently debate in the literature on their possible systematic origin. However, for completeness we refer to all signals we could identify in the literature including also references that dispute their physical origin.

\subsection{Signals in SnIa Data}

Arguably the best known tension of $\Lambda C D M$ is the difference in the value of the Hubble constant $H_{0}$ measured from two independent robust sources: local measurements using standard candles and the distance ladder and measurements using the sound horizon at recombination as a standard ruler calibrated using the $\mathrm{CMB}$ anisotropy spectrum or the Big Bang Nucleosynthesis (BBN). The locally measured value of $H_{0}$ was found to be in approximately $4-5 \sigma$ tension with the Planck18 CMB value [2,15-19]. This could be an indication of early dark energy [20] or late phantom dark energy [21,22].

Another non-standard signal that seems to exist within the SnIa data (e.g., Pantheon) is the abnormal oscillations of the $H(z)$ best fit parameter values (e.g., $\Omega_{0 m}$ ) obtained from redshift bins of the data, with respect to the corresponding best fit values of the complete dataset. This oscillating behaviour approaches the $2 \sigma$ level for low $z$ redshift bins [23-27].

This type of behaviour could be evidence of a dark energy parametrization with a similarly oscillating density, induced by a scalar field potential with a local minimum. The presence of undetected large scale inhomogeneities at low redshifts such as superclusters or voids $[28,29]$ could also provide a viable physical explanation of this phenomenon.

\subsection{Signals in the CMB Data}

A plethora of such signals, that could be either effects of systematics or indications of physical extensions of the $\Lambda$ CDM model, have been dicovered in the CMB data. The most significant of these signals are the following:

- $\quad$ The Planck CMB anisotropy power spectrum data appear to favor a universe with mildly positive curvature (a closed universe) at a $2-3 \sigma$ level. This trend is connected with the lensing anomaly and the high-low 1 tension discussed below and may represent a particular interpretation of the same signal in the CMB data [30-32].

- An anomalously strong ISW effect on scales larger than $100 \mathrm{~h}^{-1} \mathrm{Mpc}$ has been identified in the CMB data $[33,34]$. Specifically a combination with BOSS data shows a large ISW signal of supervoids with $A_{I S W} \approx 5.2 \pm 1.6$. This is in $2.6 \sigma$ tension with $\Lambda$ CDM .

- $\quad$ The CMB Cold Spot is a region of the CMB sky with scale of about $5^{\circ}$ which is unexpectedly large and cold relative in the context of the expected Gaussian CMB fluctuations. The Cold spot is approximately $70 \mu \mathrm{K}$ colder than the average CMB temperature, while the typical rms temperature variation is only $18 \mu \mathrm{K}$ [35].

- The hemispherical temperature variance asymmetry [36-38]: The CMB full-sky temperature pixels manifest a hemispherical asymmetry in power with pole axis nearly 
aligned with the Ecliptic. The northern ecliptic hemisphere is has abnormaly low variance compared to the predictions of Gaussian $\Lambda$ CDM fluctuations while the southern hemisphere is well consistent with the expected level of variance. The possible extension of this effect in polarization pixels is expected to be tested by the CMB-S4 mission [39].

- The lack of large-angle CMB temperature correlations [40]: The magnitude of the two-point angular-correlation function of the $\mathrm{CMB}$ temperature anisotropies is anomalously low for angular scales larger than about 60 degrees. Physical mechanisms operating close to the time of recombination are expected to play a role in the explanation of this observed lack of large-angle CMB temperature correlations.

- The lensing anomaly [41]: Oscillatory residuals between the Planck temperature power spectra and the best-fit $\Lambda \mathrm{CDM}$ model in the multipole range $l \in[900,1700]$ in opposite phase compared to the $\mathrm{CMB}$ and thus phenomenologically similar to the effects of gravitational lensing. This smoothing of the acoustic peaks in the temperature power spectrum could be induced by an oscillatory feature, generated during inflation [42].

- $\quad$ The preference for odd parity correlations $[43,44]$ : There is an anomalous power excess of odd $l$ multipoles compared to even $l$ multipoles in the CMB anisotropy spectrum. The odd-parity preference at low multipoles could be a phenomenological origin of the lack of large-scale CMB temperature correlation.

- $\quad$ The high-low 1 tension [45]. The $\Lambda$ CDM parameter values derived by the high $l$ part of the CMB anisotropy spectrum $(l>1000)$ are in $2-3 \sigma$ tension with the corresponding values of these parameters derived from the low $l$ part of the spectrum $(l<1000)$. This anomaly is probably related to the lensing anomaly and the indications for a closed universe discussed above.

\subsection{Signal in the Weak Leansing-RSD Data}

The low $\Omega_{0 m}-\sigma_{8}$ tension ( $S_{8}$ or growth tension. [46-53]): The value of $S_{8} \equiv \sigma_{8}\left(\Omega_{0 m} / 0.3\right)^{0.5}$ is found by weak lensing and redshift space distortion (RSD) data to be lower compared to the Planck18 value at a level of about $3 \sigma$. This indicates that dynamical cosmological probes favor lower values of $\Omega_{0 m}$ than geometric probes which could be a signal of weaker gravity than the predictions of General Relativity in the context of a $\Lambda$ CDM background.

\subsection{Age of the Universe}

The oldest stars in our vicinity were created as close to the Big Bang as possible and therefore are of a similar age with the Universe. This characteristic makes them powerful assets in determining that age. Most significantly, even a single old enough star is able to provide us with an accurate measurement. The determination of the ages of the oldest stars in our galaxy is made by using their distances by direct parallax measurements [54], as well as spectroscopic determinations of their chemical composition.

The age of the universe as obtained from local measurements using the ages of oldest stars in the Milky Way appears to be larger, and in some tension with the corresponding age obtained using the CMB Planck data in the context of $\Lambda$ CDM [55].

\subsection{Cosmic Dipoles}

There have been claims for signals indicating the violation of the cosmological principle. A physical mechanism for producing such violation on Hubble scales is studied in ref. [56]. Such signals include the following:

- The fine structure constant $\alpha$ dipole. Spectra from quasars indicate a spatially dependent value of the fine structure constant at a $4 \sigma$ level of significance. This signal indicates both the violation of the cosmological principle and variation of the fundamental constants $[57,58]$. This dipole is also anomalously aligned with others $[59,60]$.

- The large scale velocity flow dipole $[61,62]$. 
- The quasar density dipole, which is a statistically significant $(4 \sigma)$ dipole in the density of quasars with direction close to the CMB dipole [63].

\subsection{Signal in BAO Data}

The Lyman- $\alpha$ forest BAO anomaly (galaxy vs $L y-\alpha$ BAO) [64,65] refers to a $2.5-3 \sigma$ discrepancy between the BAO peak in the Ly- $\alpha$ forest at an effective redshift of $z \sim 2.34$ and the best fit Planck18 $\Lambda$ CDM cosmology. This abnormality was found to be present in the data even in the case where it is assumed that the BAO scale is a standard ruler independent of the sound horizon.

Since the anomaly was first reported studying the Ly- $\alpha$ forest at a redshsift of $z \sim 2.34$ it could imply evolution of the dark energy equation of state $w(z)$ in the range $0.57<z<2.34$.

\subsection{Parity Violating Rotation of CMB Linear Polarization}

A parity violating axion-like scalar field, which can play the role of dark matter and dark energy, could rotate the plane of linear polarization of CMB photons as they travel from the last scattering surface to the present by a non-zero angle $\beta$ (cosmic birefringence angle). A non-zero value of $\beta$ was recently detected the Planck18 polarization data at a $2.4 \sigma$ statistical significance level [66].

This study provides a non-zero estimate for $\beta$ with a confidence of $99.2 \%$ C.L. If proven to be correct this would be a very significant result which would hint towards new physics beyond $\Lambda \mathrm{CDM}$, sensitive to parity violation.

\subsection{The Lithium Problem}

Big Bang Nucleosynthesis (BBN) is very useful tool in cosmology since it has the rare quality of connecting the early Universe with present day observations. However, despite of the great success that the theory of BBN has in explaining the creation and abundancy of the elements observed in our Universe, it fails while trying to explain the observed quantity of Lithium. Specificaly, the observed value of Lithium is $\simeq 3.5$ smaller than that predicted by BBN $[67,68]$. In particular measurements of old, metal-poor stars in the Milky Way's halo find 5 times less lithium that BBN predicts.

\subsection{Quasar Hubble Diagram}

A possible deviation from the $\Lambda \mathrm{CDM}$ cosmology hinting towards phantom dark energy has been documented when constructing a Hubble diagram using quasars as distance indicators, in the redshift range of $0.5<z<5.5[69,70]$. The observed tension between the best fit cosmographic parameters and $\Lambda \mathrm{CDM}$ could reach $4 \sigma$, even when combining the quasar data with the usual SnIa datasets.

This deviation from $\Lambda \mathrm{CDM}$ seems at first glance to be a genuine tension, however, a strong case can be made towards the opposite [71]. Specifically, it could be argued that the log-polynomial expansion of the luminosity distance relation,

$$
d_{L}(z)=\frac{\operatorname{cln}(10)}{H_{0}}\left[\log _{10}(1+z)+a_{2} \log _{10}^{2}(1+z)+a_{3} \log _{10}^{3}(1+z)+\ldots\right]
$$

where $H_{0}, a_{2}, a_{3}, \ldots$ are free parameters, used to construct the aforementioned diagram is not valid for redshifts larger than 2, a fact that points towards the observed tension being an artifact.

\subsection{Oscillating Force Signals in Short Range Gravity Experiments}

A re-analysis of short range gravity experiments has indicated the presence of an oscillating force signal with sub-mm wavelength at a $2 \sigma$ level [72,73]. This type of signal seems to hold some statistical significance and could be hint towards several possible physical effects, amongst them an indication for a short distance modification of GR.

This oscillating behaviour could be seen as evidence for emerging signatures of nonlocal behaviour in experimental data. It could also provide a motivation for re-examining 
the stability of $f(R)$ gravity with negative squared mass which are thought to be unstable at the perturbative level.

\section{Conclusions and Discussion}

The signals discussed in this contribution, as well as others not covered, could be interpreted as telltale signs of the need to incorporate a model more complex than $\Lambda$ CDM as the new standard model of Cosmology. There arises, therefore, the need to investigate new fundamental physics with the aim to reconcile the emerging tensions.

Such interesting new physics, is most likely to affect four basic observable parameters: The Hubble parameter $H(z, w)$, the effective Newton constants for growth of perturbations $\mu \equiv \frac{G_{e f f}}{G_{N}}$ and lensing $\Sigma \equiv \frac{G_{L}}{G_{N}}$, as well as the fine structure constant $\alpha$ ( $w$ is the dark energy equation of state parameter and $G_{N}$ is the locally measured value of the Newton's constant). According to $\Lambda \mathrm{CDM} H(z)=H(z, w=-1), \mu=1, \Sigma=1$. The fine structure constant $\alpha$ is also assumed constant and uniform in the standard model.

Generic extensions of $\Lambda C D M$ may allow for a redshift dependence of the parameters $w, \mu, \Sigma$ and $\alpha$ as well as a possible large scale spatial dependence which could violate the cosmological principle. Varying fundamental constants can potentially address the fine structure constant $\alpha$ dipole, the lithium problem, growth tension, SnIa signals (variation of the SnIa absolute magnitude $\mathcal{M}$ ), quasar signals and the ISW CMB signal.

The identification of the new physics that can explain the nonstandard signals detected in the data can be realized in an effective manner through the following strategy:

- Tuning of current missions towards the verification or rejection of non-standard signals.

- Identification of favored parametrizations of $H(z, w(z), r), \mu(z, r), \Sigma(z, r), \alpha(z, r)$ assuming that at least some of the non-standard signals are physical.

- Identification of the theoretical models (field Lagrangians) that are consistent with these parametrizations. Interestingly, for example only a small subset of modified gravity models is consistent with the weak gravity in the context of a $\Lambda$ CDM background [74-77] suggested in the context of the $S_{8}$ tension.

In view of the upcoming volume of emerging new cosmological data in the next decade, it is likely that the observed nonstandard cosmological signal will be translated into exciting new physical theories.

Funding: This research is co-financed by Greece and the European Union (European Social FundESF) through the Operational Programme "Human Resources Development, Education and Lifelong Learning 2014-2020" in the context of the project "Scalar fields in Curved Spacetimes: Soliton Solutions, Observational Results and Gravitational Waves" (MIS 5047648).

\section{References}

1. Ade, P.A.; Aghanim, N.; Arnaud, M.; Ashdown, M.; Aumont, J.; Baccigalupi, C.; Banday, A.J.; Barreiro, R.B.; Bartlett, J.G.; Bartolo, N.; et al. Planck 2015 results. XIII. Cosmological parameters. Astron. Astrophys. 2016, 594, A13. [CrossRef]

2. Aghanim, N.; Akrami, Y.; Ashdown, M.; Aumont, J.; Baccigalupi, C.; Ballardini, M.; Banday, A.J.; Barreiro, R.B.; Bartolo, N.; Basak, S.; et al. Planck 2018 results. VI. Cosmological parameters. Astron. Astrophys. 2020, 641, A6. [CrossRef]

3. Betoule, M.E.A.; Kessler, R.; Guy, J.; Mosher, J.; Hardin, D.; Biswas, R.; Astier, P.; El-Hage, P.; Konig, M.; Kuhlmann, S.; et al. Improved cosmological constraints from a joint analysis of the SDSS-II and SNLS supernova samples. Astron. Astrophys. 2014, 568, A22. [CrossRef]

4. Scolnic, D.M.; Jones, D.O.; Rest, A.; Pan, Y.C.; Chornock, R.; Foley, R.J.; Huber, M.E.; Kessler, R.; Narayan, G.; Riess, A.G. The Complete Light-curve Sample of Spectroscopically Confirmed SNe Ia from Pan-STARRS1 and Cosmological Constraints from the Combined Pantheon Sample. Astrophys. J. 2018, 859, 101. [CrossRef]

5. Gómez-Valent, A.; Amendola, L. $H_{0}$ from cosmic chronometers and Type Ia supernovae, with Gaussian Processes and the novel Weighted Polynomial Regression method. JCAP 2018, 04, 051. [CrossRef]

6. Alam, S.; Ata, M.; Bailey, S.; Beutler, F.; Bizyaev, D.; Blazek, J.A.; Bolton, A.S.; Brownstein, J.R.; Burden, A.; Chuang, C.-H.; et al. The clustering of galaxies in the completed SDSS-III Baryon Oscillation Spectroscopic Survey: Cosmological analysis of the DR12 galaxy sample. Mon. Not. R. Astron. Soc. 2017, 470, 2617-2652. [CrossRef]

7. Aubourg, É.; Bailey, S.; Bautista, J.E.; Beutler, F.; Bhardwaj, V.; Bizyaev, D.; Blanton, M.; Blomqvist, M.; Bolton, A.S.; Bovy, J.; et al. Cosmological implications of baryon acoustic oscillation measurements. Phys. Rev. D 2015, 92, 123516. [CrossRef] 
8. Baxter, E.; Clampitt, J.; Giannantonio, T.; Dodelson, S.; Jain, B.; Huterer, D.; Bleem, L.; Crawford, T.; Efstathiou, G.; Fosalba, P.; et al. Joint measurement of lensing-galaxy correlations using SPT and DES SV data. Mon. Not. R. Astron. Soc. 2016, 461, $4099-4114$. [CrossRef]

9. Efstathiou, G.; Lemos, P. Statistical inconsistencies in the KiDS-450 data set. Mon. Not. R. Astron. Soc. 2018, 476, 151-157. [CrossRef]

10. Rozo, E.; Wechsler, R.; Rykoff, E.S.; Annis, J.T.; Becker, M.; Evrard, A.E.; Frieman, J.A.; Hansen, S.M.; Hao, J.; Johnston, D.E.; et al. Cosmological Constraints from the SDSS maxBCG Cluster Catalog. Astrophys. J. 2010, 708, 645-660. [CrossRef]

11. Basilakos, S.; Nesseris, S. Testing Einstein's gravity and dark energy with growth of matter perturbations: Indications for new physics? Phys. Rev. D 2016, 94, 123525. [CrossRef]

12. Quelle, A.; Maroto, A.L. On the tension between growth rate and CMB data. Eur. Phys. J. C 2020, 80, 369. [CrossRef]

13. Perivolaropoulos, L. Six Puzzles for LCDM Cosmology. arXiv 2008, arXiv:0811.4684.

14. Perivolaropoulos, L. LCDM: Triumphs, Puzzles and Remedies. J. Cosmol. 2011, 15, 6054.

15. Riess, A.G.; Macri, L.M.; Hoffmann, S.L.; Scolnic, D.; Casertano, S.; Filippenko, A.V.; Tucker, B.E.; Reid, M.J.; Jones, D.O.; Silverman, J.M.; et al. A 2.4\% Determination of the Local Value of the Hubble Constant. Astrophys. J. 2016, 826, 56. [CrossRef]

16. Riess, A.G.; Yuan, W.; Casertano, S.; Macri, L.M.; Scolnic, D. The Accuracy of the Hubble Constant Measurement Verified through Cepheid Amplitudes. Astrophys. J. Lett. 2020, 896, L43. [CrossRef]

17. Verde, L.; Treu, T.; Riess, A.G. Tensions between the Early and the Late Universe. Nat. Astron. 2019, 3, 891. [CrossRef]

18. Di Valentino, E.; Melchiorri, A.; Silk, J. Cosmological constraints in extended parameter space from the Planck 2018 Legacy release. JCAP 2020, 01, 013. [CrossRef]

19. Renzi, F.; Silvestri, A. A look at the Hubble speed from first principles. arXiv 2020, arXiv:2011.10559.

20. Chudaykin, A.; Gorbunov, D.; Nedelko, N. Combined analysis of Planck and SPTPol data favors the early dark energy models. JCAP 2020, 08, 013. [CrossRef]

21. Alestas, G.; Kazantzidis, L.; Perivolaropoulos, L. $H_{0}$ tension, phantom dark energy, and cosmological parameter degeneracies. Phys. Rev. D 2020, 101, 123516. [CrossRef]

22. Alestas, G.; Kazantzidis, L.; Perivolaropoulos, L. A $w$ phantom transition at $z_{t}<0.1$ as a resolution of the Hubble tension. Phys. Rev. D 2021, 103, 083517.

23. Riess, A.G.; Rodney, S.A.; Scolnic, D.M.; Shafer, D.L.; Strolger, L.-G.; Ferguson, H.C.; Postman, M.; Graur, O.; Maoz, D.; Jha, S.W.; et al. Type Ia Supernova Distances at Redshift $>1.5$ from the Hubble Space Telescope Multi-cycle Treasury Programs: The Early Expansion Rate. Astrophys. J. 2018, 853, 126, [CrossRef]

24. Colgáin, E.O. A hint of matter underdensity at low z? JCAP 2019, 09, 006. [CrossRef]

25. Camarena, D.; Marra, V. Local determination of the Hubble constant and the deceleration parameter. Phys. Rev. Res. 2020, 2, 013028. [CrossRef]

26. Kazantzidis, L.; Koo, H.; Nesseris, S.; Perivolaropoulos, L.; Shafieloo, A. Hints for possible low redshift oscillation around the best fit $\Lambda$ CDM model in the expansion history of the universe. Mon. Not. R. Astron. Soc. 2021, 501, 3421-3426. [CrossRef]

27. Kazantzidis, L.; Perivolaropoulos, L. Hints of a Local Matter Underdensity or Modified Gravity in the Low $z$ Pantheon data. Phys. Rev. D 2020, 102, 023520. [CrossRef]

28. Shanks, T.; Hogarth, L.; Metcalfe, N. Gaia Cepheid parallaxes and 'Local Hole' relieve $H_{0}$ tension. Mon. Not. R. Astron. Soc. 2019, 484, L64-L68. [CrossRef]

29. Grande, J.; Perivolaropoulos, L. Generalized LTB model with Inhomogeneous Isotropic Dark Energy: Observational Constraints. Phys. Rev. D 2011, 84, 023514. [CrossRef]

30. Di Valentino, E.; Anchordoqui, L.A.; Akarsu, Ö; Ali-Haimoud, Y.; Amendola, L.; Arendse, N.; Asgari, M.; Ballardini, M.; Basilakos, S.; Battistelli, E.; et al. Cosmology Intertwined IV: The Age of the Universe and its Curvature. Astropart. Phys. 2021, $131,102607$. [CrossRef]

31. Handley, W. Curvature tension: Evidence for a closed universe. Phys. Rev. D 2021, 103, L041301. [CrossRef]

32. Vagnozzi, S.; Di Valentino, E.; Gariazzo, S.; Melchiorri, A.; Mena, O.; Silk, J. Listening to the BOSS: the galaxy power spectrum take on spatial curvature and cosmic concordance. arXiv 2020, arXiv:2010.02230.

33. Granett, B.R.; Neyrinck, M.C.; Szapudi, I. An Imprint of Super-Structures on the Microwave Background due to the Integrated Sachs-Wolfe Effect. Astrophys. J. Lett. 2008, 683, L99-L102. [CrossRef]

34. Kovács, A.; Sánchez, C.; Garcia-Bellido, J.; Elvin-Poole, J.; Hamaus, N.; Miranda, V.; Nadathur, S.; Abbott, T.; Abdalla, F.B.; Annis, J.; et al. More out of less: An excess integrated Sachs-Wolfe signal from supervoids mapped out by the Dark Energy Survey. Mon. Not. R. Astron. Soc. 2019, 484, 5267-5277. [CrossRef]

35. Cruz, M.; Cayon, L.; Martinez-Gonzalez, E.; Vielva, P.; Jin, J. The non-gaussian cold spot in the 3-year wmap data. Astrophys. J. 2007, 655, 11-20. [CrossRef]

36. Monteserin, C.; Barreiro, R.B.B.; Vielva, P.; Martinez-Gonzalez, E.; Hobson, M.P.; Lasenby, A.N. A low CMB variance in the WMAP data. Mon. Not. R. Astron. Soc. 2008, 387, 209-219. [CrossRef]

37. Planck Collaboration; Ade, P.; Arnaud, M.; Ashdown, M.; Aumont, J.; Baccigalupi, C.; Banday, A.J.; Barreiro, R.B.; Battaner, E.; Benabed, K.; et al. Planck 2013 results. XXXI. Consistency of the Planck data. Astron. Astrophys. 2014, 571, A31. [CrossRef]

38. O’Dwyer, M.; Copi, C.J.; Nagy, J.M.; Netterfield, C.B.; Ruhl, J.; Starkman, G.D. Hemispherical variance anomaly and reionization optical depth. Mon. Not. R. Astron. Soc. 2020, 499, 3563-3570. [CrossRef] 
39. O'Dwyer, M.; Copi, C.J.; Knox, L.; Starkman, G.D. CMB-S4 and the Hemispherical Variance Anomaly. Mon. Not. R. Astron. Soc. 2017, 470, 372-378. [CrossRef]

40. Copi, C.J.; Huterer, D.; Schwarz, D.J.; Starkman, G.D. Lack of large-angle TT correlations persists in WMAP and Planck. Mon. Not. R. Astron. Soc. 2015, 451, 2978-2985. [CrossRef]

41. Motloch, P.; Hu, W. Lensinglike tensions in the Planck legacy release. Phys. Rev. D 2020, 101, 083515. [CrossRef]

42. Domènech, G.; Kamionkowski, M. Lensing anomaly and oscillations in the primordial power spectrum. JCAP 2019, 11, 040. [CrossRef]

43. Kim, J.; Naselsky, P. Anomalous parity asymmetry of WMAP power spectrum data at low multpoles: Is it cosmological or systematics? Phys. Rev. D 2010, 82, 063002. [CrossRef]

44. Kim, J.; Naselsky, P. Lack of angular correlation and odd-parity preference in cosmic microwave background data. Astrophys. J. 2011, 739, 79. [CrossRef]

45. Addison, G.E.; Huang, Y.; Watts, D.J.; Bennett, C.L.; Halpern, M.; Hinshaw, G.; Weiland, J.L. Quantifying discordance in the 2015 Planck CMB spectrum. Astrophys. J. 2016, 818, 132. [CrossRef]

46. Asgari, M.; Tröster, T.; Heymans, C.; Hildebrandt, H.; Busch, J.L.V.D.; Wright, A.H.; Choi, A.; Erben, T.; Joachimi, B.; Joudaki, S.; et al. KiDS+VIKING-450 and DES-Y1 combined: Mitigating baryon feedback uncertainty with COSEBIs. Astron. Astrophys. 2020, 634, A127. [CrossRef]

47. Abbott, T.M.C.; Aguena, M.; Alarcon, A.; Allam, S.; Allen, S.; Annis, J.; Avila, S.; Bacon, D.; Bechtol, K.; Bermeo, A.; et al. Dark Energy Survey Year 1 Results: Cosmological constraints from cluster abundances and weak lensing. Phys. Rev. D 2020, 102, 023509. [CrossRef]

48. Macaulay, E.; Wehus, I.K.; Eriksen, H.K. Lower Growth Rate from Recent Redshift Space Distortion Measurements than Expected from Planck. Phys. Rev. Lett. 2013, 111, 161301. [CrossRef]

49. Skara, F.; Perivolaropoulos, L. Tension of the $E_{G}$ statistic and redshift space distortion data with the Planck- $\Lambda C D M$ model and implications for weakening gravity. Phys. Rev. D 2020, 101, 063521. [CrossRef]

50. Kazantzidis, L.; Perivolaropoulos, L. Is gravity getting weaker at low z? Observational evidence and theoretical implications. arXiv 2019, arXiv:1907.03176.

51. Bull, P.; Akrami, Y.; Adamek, J.; Baker, T.; Bellini, E.; Jiménez, J.B.; Bentivegna, E.; Camera, S.; Clesse, S.; Davis, J.; et al. Beyond $\Lambda$ CDM: Problems, solutions, and the road ahead. Phys. Dark Univ. 2016, 12, 56-99. [CrossRef]

52. Kazantzidis, L.; Perivolaropoulos, L. Evolution of the $f \sigma_{8}$ tension with the Planck $15 / \Lambda C D M$ determination and implications for modified gravity theories. Phys. Rev. D 2018, 97, 103503. [CrossRef]

53. Nesseris, S.; Pantazis, G.; Perivolaropoulos, L. Tension and constraints on modified gravity parametrizations of $G_{\mathrm{eff}}(z)$ from growth rate and Planck data. Phys. Rev. D 2017, 96, 023542. [CrossRef]

54. Luri, X.; Brown, A.G.A.; Sarro, L.M.; Arenou, F.; Bailer-Jones, C.A.L.; Castro-Ginard, A.; de Bruijne, J.; Prusti, T.; Babusiaux, C.; Delgado, H.E. Gaia Data Release 2-Using Gaia parallaxes. Astron. Astrophys. 2018, 616, A9. [CrossRef]

55. Verde, L.; Protopapas, P.; Jimenez, R. Planck and the local Universe: Quantifying the tension. Phys. Dark Univ. 2013, 2, 166-175. [CrossRef]

56. Bueno Sanchez, J.C.; Perivolaropoulos, L. Topological Quintessence. Phys. Rev. D 2011, 84, 123516. [CrossRef]

57. Dumont, V.; Webb, J.K. Modelling long-range wavelength distortions in quasar absorption echelle spectra. Mon. Not. R. Astron. Soc. 2017, 468, 1568-1574. [CrossRef]

58. Webb, J.K.; King, J.A.; Murphy, M.T.; Flambaum, V.V.; Carswell, R.F.; Bainbridge, M.B. Indications of a spatial variation of the fine structure constant. Phys. Rev. Lett. 2011, 107, 191101. [CrossRef]

59. Mariano, A.; Perivolaropoulos, L. Is there correlation between Fine Structure and Dark Energy Cosmic Dipoles? Phys. Rev. D 2012, 86, 083517. [CrossRef]

60. Mariano, A.; Perivolaropoulos, L. CMB Maximum temperature asymmetry Axis: Alignment with other cosmic asymmetries. Phys. Rev. D 2013, 87, 043511. [CrossRef]

61. Wiltshire, D.L.; Smale, P.R.; Mattsson, T.; Watkins, R. Hubble flow variance and the cosmic rest frame. Phys. Rev. D 2013, 88, 083529. [CrossRef]

62. Bengaly, C.A.P.; Maartens, R.; Santos, M.G. Probing the Cosmological Principle in the counts of radio galaxies at different frequencies. JCAP 2018, 04, 031. [CrossRef]

63. Secrest, N.J.; von Hausegger, S.; Rameez, M.; Mohayaee, R.; Sarkar, S.; Colin, J. A Test of the Cosmological Principle with Quasars Astrophys. J. 2021, 908, L51. [CrossRef]

64. Cuceu, A.; Farr, J.; Lemos, P.; Font-Ribera, A. Baryon Acoustic Oscillations and the Hubble Constant: Past, Present and Future. JCAP 2019, 10, 044. [CrossRef]

65. Evslin, J. Isolating the Lyman Alpha Forest BAO Anomaly. JCAP 2017, 04, 024. [CrossRef]

66. Minami, Y.; Komatsu, E. New Extraction of the Cosmic Birefringence from the Planck 2018 Polarization Data. Phys. Rev. Lett. 2020, 125, 221301. [CrossRef] [PubMed]

67. Cyburt, R.H.; Fields, B.D.; Olive, K.A. An Update on the big bang nucleosynthesis prediction for Li-7: The problem worsens. JCAP 2008, 11, 012. [CrossRef]

68. Clara, M.T.; Martins, C.J.A.P. Primordial nucleosynthesis with varying fundamental constants: Improved constraints and a possible solution to the Lithium problem. Astron. Astrophys. 2020, 633, L11. [CrossRef] 
69. Risaliti, G.; Lusso, E. Cosmological constraints from the Hubble diagram of quasars at high redshifts. Nat. Astron. $2019,3,272-277$. [CrossRef]

70. Lusso, E.; Piedipalumbo, E.; Risaliti, G.; Paolillo, M.; Bisogni, S.; Nardini, E.; Amati, L. Tension with the flat $\Lambda$ CDM model from a high-redshift Hubble diagram of supernovae, quasars, and gamma-ray bursts. Astron. Astrophys. 2019, 628, L4. [CrossRef]

71. Banerjee, A.; Colgáin, E.O.; Sasaki, M.; Sheikh-Jabbari, M.M.; Yang, T. On cosmography in the cosmic dark ages: Are we still in the dark? arXiv 2020, arXiv:2009.04109.

72. Perivolaropoulos, L. Submillimeter spatial oscillations of Newton's constant: Theoretical models and laboratory tests. Phys. Rev. D 2017, 95, 084050. [CrossRef]

73. Antoniou, I.; Perivolaropoulos, L. Constraints on spatially oscillating sub-mm forces from the Stanford Optically Levitated Microsphere Experiment data. Phys. Rev. D 2017, 96, 104002. [CrossRef]

74. Wittner, M.; Laverda, G.; Piattella, O.F.; Amendola, L. Transient weak gravity in scalar-tensor theories. JCAP 2020, $07,019$. [CrossRef]

75. Pizzuti, L.; Saltas, I.D.; Casas, S.; Amendola, L.; Biviano, A. Future constraints on the gravitational slip with the mass profiles of galaxy clusters. Mon. Not. R. Astron. Soc. 2019, 486, 596-607. [CrossRef]

76. Gannouji, R.; Perivolaropoulos, L.; Polarski, D.; Skara, F. Weak gravity on a $\Lambda$ CDM background Phys. Rev. D 2021, 103, 063509. [CrossRef]

77. Gannouji, R.; Kazantzidis, L.; Perivolaropoulos, L.; Polarski, D. Consistency of modified gravity with a decreasing $G_{\text {eff }}(z)$ in a $\Lambda$ CDM background. Phys. Rev. D 2018, 98, 104044. [CrossRef] 